\title{
ON SOME DOUBLE LACUNARY SEQUENCE SPACES OF FUZZY NUMBERS
}

\author{
Ekrem Savaş \\ Istanbul Commerce University \\ Department of Mathematics \\ Üsküdar-Istanbul, TURKEY \\ esavas@iticu.edu.tr
}

\begin{abstract}
In this paper we introduce a new concept for lacunary strong P-convergent with respect to an Orlicz function and examine some properties of the resulting sequence space of fuzzy numbers. We also show that if a sequence is lacunary strong Pconvergence with respect to an Orlicz function then it is $S_{\theta_{r, s}}(F)$-convergent.
\end{abstract}

Key Words: Orlicz function, Double lacunary sequences, P-convergent, Fuzzy numbers

\section{INTRODUCTION}

Among various developments of the theory of fuzzy sets [28] a progressive development has been made to find the fuzzy analogues of the classical set theory. In fact the fuzzy theory has become an area of active research for the last 40 years. It has wide range applications in the field of science and engineering, e.g., population dynamics [1], chaos control [8], computer programming [9], nonlinear dynamical systems [10], fuzzy physics [13], fuzzy topology [22], etc. Recently fuzzy topology proves to be a very useful tool to deal with such situation where the use of classical theories breaks down. The most fascinating application of fuzzy topology in quantum particle physics arises in string and $\varepsilon^{\infty}$-theory of El-Nashie $[4,5,6]$.

In [18], Nanda studied on sequences of fuzzy numbers and showed that the set of all convergent sequences of fuzzy numbers forms a complete metric space. Nuray [19] proved the inclusion relations between the set of statistically convergent and lacunary statistically convergent sequences of fuzzy numbers. Savas [23] introduced and discussed double convergent sequences of fuzzy numbers and showed that the set of all double convergent sequences of fuzzy numbers is complete.

The idea of statistical convergence of a sequence was introduced by Fast [7]. Statistical convergence was generalized by Buck [2] and studied by other authors, using a regular nonnegative summability matrix $A$ in place of Cesáro matrix. The existing literature on double statistical convergence appears to have been restricted to real or complex sequences, but at the first time Savaş and Mursaleen [26] extended the idea to apply to double sequences of fuzzy numbers.

The study of Orlicz sequence spaces was initiated with a certain specific purpose in Banach space theory. Lindenstrauss and Tzafriri [14] investigated Orlicz sequence spaces in 
more detail, and they proved that every Orlicz sequence space $l_{M}$ contains a subspace isomorphic to $l_{p}(1 \leq p<\infty)$. The Orlicz sequence spaces are the special cases of Orlicz spaces studied in [12]. Orlicz spaces find a number of useful applications in the theory of nonlinear integral equations. Whereas the Orlicz sequence spaces are the generalization of $l_{p}$-spaces, the $l_{p}$-spaces find themselves enveloped in Orlicz spaces [11].

Subsequently the notion of Orlicz function was used to define sequence spaces by Parashar and Choudhary [20] and other authors. An Orlicz function $M$ can be represented in the following integral form:

$$
M(x)=\int_{0}^{x} p(t) d t
$$

where $p$ is the known kernel of $M$, right differential for $t \geq 0, p(0)=0, p(t)>0$ for $t>0, p$ is non-decreasing and $p(t) \rightarrow \infty$ as $t \rightarrow \infty$. Recently Savas generalized $c(\Delta)$ and $l_{\infty}(\Delta)$ by using Orlicz function and also established some inclusion theorems.

In this paper, using an Orlicz function some sequence spaces of fuzzy numbers have been given. Later on we give a brief overview about fuzzy numbers, Orlicz function and also we define the concepts of double lacunary sequence spaces of fuzzy numbers with respect to the Orlicz function $M$ and also we prove some inclusion relations between $\left[N_{\theta_{r, s}}, M\right]^{F}$ and $S_{\theta_{r, s}}(F)$.

\section{INTRODUCTION AND BACKGROUND}

We begin this definition presentation with the statement of a few preliminaries which are useful in the sequel of this paper.

Recall in [12] that an Orlicz function $M:[0, \infty) \rightarrow[0, \infty)$ is continuous, convex, non decreasing function define for $x>0$ such that $M(0)=0$ and $M(x)>0$ for $x>0$, and $M(x) \rightarrow \infty$ as $x \rightarrow \infty$.

Let $C\left(R^{n}\right)=\left\{A \subset R^{n}: A\right.$ compact and convex $\}$. The spaces $C\left(R^{n}\right)$ has a linear structure induced by the operations

$$
A+B=\{a+b: a \in A, b \in B\}
$$

and

$$
\lambda A=\{\lambda a: \lambda \in A\}
$$

for $A, B \in C\left(R^{n}\right)$ and $\lambda \in R$. The Hausdorff distance between $A$ and $B$ of $C\left(R^{n}\right)$ is defined as

$$
\delta_{\infty}(A, B)=\max \left\{\operatorname{supinf}_{a \in A}\|a-b\|, \sup _{b \in B} \inf _{a \in A}\|a-b\|\right\}
$$

where $\|\cdot\|$ denotes the usual Euclidean norm in $R^{n}$. It is well known that $\left(C\left(R^{n}\right), \delta_{\infty}\right)$ is a complete (not separable) metric space. 
A fuzzy number is a function $X$ from $R^{n}$ to $[0,1]$ satisfying

(1) $X$ is normal, i.e. there exists an $x_{0} \in R^{n}$ such that $X\left(x_{0}\right)=1$;

(2) $X$ is fuzzy convex, i.e. for any $x, y \in R^{n}$ and $0 \leq \lambda \leq 1$,

$$
X(\lambda x+(1-\lambda) y) \geq \min \{X(x), X(y)\}
$$

(3) $X$ is upper semi-continuous;

(4) The closure of $\left\{x \in R^{n}: X(x)>0\right\}$; denoted by $X^{0}$, is compact.

These properties imply that for each $0<\alpha \leq 1$, the $\alpha$-level set

$$
X^{\alpha}=\left\{x \in R^{n}: X(x) \geq \alpha\right\}
$$

is a nonempty compact convex, subset of $R^{n}$, as is the support $X^{0}$. Let $L\left(R^{n}\right)$ denote the set of all fuzzy numbers. The linear structure of $L\left(R^{n}\right)$ induces addition $X+Y$ and scalar multiplication , $\lambda X, \lambda \in R$, in terms of $\alpha$-level sets, by

$$
[X+Y]^{\alpha}=[X]^{\alpha}+[Y]^{\alpha}
$$

and

$$
[\lambda X]^{\alpha}=\lambda[X]^{\alpha}
$$

for each $0 \leq \alpha \leq 1$.

Define for each $1 \leq q<\infty$

$$
d_{q}(X, Y)=\left\{\int_{0}^{1} \delta_{\infty}\left(X^{\alpha}, Y^{\alpha}\right)^{q} d \alpha\right\}^{1 / q}
$$

and $d_{\infty}(X, Y)=\sup _{0 \leq \alpha \leq 1} \delta_{\infty}\left(X^{\alpha}, Y^{\alpha}\right)$ clearly $d_{\infty}(X, Y)=\lim _{q \rightarrow \infty} d_{q}(X, Y)$ with $d_{q} \leq d_{r}$ if $q \leq r$. Moreover $d_{q}$ is a complete, separable and locally compact metric space [3].

Throughout the paper, $d$ will denote $d_{q}$ with $1 \leq q<\infty$.

Definition 2.1. The double sequence $\theta_{r, s}=\left\{k_{r}, l_{s}\right\}$ is called double lacunary if there exist two increasing sequences of integers such that

$$
k_{0}=0, h_{r}=k_{r}-k_{r-1} \rightarrow \infty \text { as } r \rightarrow \infty
$$

and

$$
l_{0}=0, \bar{h}_{s}=l_{s}-l_{s-1} \rightarrow \infty \text { as } s \rightarrow \infty .
$$

Let's denote $k_{r, s}=k_{r} l_{s}, h_{r, s}=h_{r} \bar{h}_{s}$ and $\theta_{r, s}$ is determine by $I_{r, s}=\left\{(k, l): k_{r-1}<k \leq k_{r} \& l_{s-1}<l \leq l_{s}\right\}, q_{r}=\frac{k_{r}}{k_{r-1}}, \bar{q}_{s}=\frac{l_{s}}{l_{s-1}}$ and $q_{r, s}=q_{r} \bar{q}_{s}$.

We have the following definitions. 
Definition 2.2. A double sequence $X=\left(X_{k l}\right)$ of fuzzy numbers is said to be convergent in the Pringsheim's sense or P-convergent to a fuzzy number $X_{0}$, iffor every $\varepsilon>0$ there exists $N \in \boldsymbol{N}$ such that

$$
d\left(X_{k l}, X_{0}\right)<\varepsilon \text { for } k, l>N
$$

where $\boldsymbol{N}$ is the set of natural numbers, and we denote by $P-\lim X=X_{0}$. The number $X_{0}$ is called the Pringsheim limit of $X_{k l}$.

More exactly we say that a double sequence $\left(X_{k l}\right)$ converges to a finite number $X_{0}$ if $X_{k l}$ tend to $X_{0}$ as both $k$ and l tends to $\infty$ independently of one another.

Let $c^{2}(F)$ denote the set of all double P-convergent sequences of fuzzy numbers.

Definition 2.3. Let $p=\left(p_{k, l}\right)$ be any factorable double sequence of positive real numbers. The double sequence $X=\left(X_{k, l}\right)$ is strongly p-Cesáro summable with respect to the Orlicz function $M$ if

$$
\left[\sigma_{1,1}, M, p\right]^{F}:=\left\{X=\left(X_{k, l}\right): P-\lim _{m n} \frac{1}{m, n} \sum_{k, l=1,1}^{m, n}\left[M\left(\frac{d\left(X_{k, l}, X_{0}\right.}{\rho}\right)\right]^{p_{k, l}}=0\right\} .
$$

Definition 2.4. Let $M$ be an Orlicz function and $p=\left(p_{k, l}\right)$ be any factorable double sequence of positive real numbers, we define the following sequence spaces:

$$
\begin{aligned}
& {\left[N_{\theta_{r, s}}, M, p\right]^{F}=\left\{X=\left(X_{k, l}\right): P-\lim _{r, s} \frac{1}{h_{r s}} \sum_{(k, l) \in I_{r, s}}\left[M\left(\frac{d\left(X_{k, l}, X_{0}\right.}{\rho}\right)\right]^{p_{k, l}}=0, \text { for some } \rho>0\right\},} \\
& {\left[N_{\theta_{r, s}}, M, p\right]_{0}^{F}=\left\{X=\left(X_{k, l}\right): P-\lim _{r, s} \frac{1}{h_{r s}} \sum_{(k, l) \in I_{r, s}}\left[M\left(\frac{d\left(X_{k, l}, \overline{0}\right.}{\rho}\right)\right]^{p_{k, l}}=0, \text { for some } \rho>0\right\},}
\end{aligned}
$$

and

$$
\left[N_{\theta_{r, s}}, M, p\right]_{\infty}^{F}=\left\{X=\left(X_{k, l}\right): \sup _{r, s} \frac{1}{h_{r s}} \sum_{(k, l) \in I_{r, s}}\left[M\left(\frac{d\left(X_{k, l}, \overline{0}\right.}{\rho}\right)\right]^{p_{k, l}}<\infty, \text { for some } \rho>0\right\}
$$

where $\overline{0}(t)=1$, if $t=(0,0, \ldots, 0)$, otherwise. We shall denote $\left[N_{\theta_{r, s}}, M, p\right]^{F}$, $\left[N_{\theta_{r, s}}, M, p\right]_{0}^{F}$ and $\left[N_{\theta_{r, s}}, M, p\right]_{\infty}^{F}$ as $\left[N_{\theta_{r, s}}, M\right]^{F},\left[N_{\theta_{r, s}}, M\right]_{0}^{F}$ and $\left[N_{\theta_{r, s}}, M\right]_{\infty}^{F}$ respectively when $p_{k, l}=1$ for all $k$ and $l$. If $X$ is in $\left[N_{\theta_{r, s}}, M\right]^{F}$ we shall say that $X$ is strongly double lacunary P-convergent with respect to the Orlicz function $M$. In this case we write $X_{k l} \rightarrow X_{0}\left[N_{\theta_{r, s}}, M\right]^{F}$. Also note if $M(X)=X$, and $p_{k, l}=1$ for all $k$ and $l$, then $\left[N_{\theta_{r, s}}, M, p\right]^{F}=N_{\theta_{r, s}}^{F}$ which are presented by Savas in [27] as follows: 


$$
N_{\theta_{r, s}}^{F}=\left\{X=\left(X_{k, l}\right): P-\lim _{r, s} \frac{1}{h_{r s}} \sum_{(k, l) \in I_{r, s}} d\left(X_{k, l}, X_{0}\right)=0\right\} .
$$

With these new concepts we can now consider the following theorem.

Theorem 2.1. If $p_{k, l}>0$ and $X$ is strongly double lacunary convergent to $X_{0}$, with respect to the Orlicz function $M$, that is $X_{k l} \rightarrow X_{0}\left[N_{\theta_{r, s}}, M, p\right]^{F}$, then $X_{0}$ is unique.

Proof. Let $\lim p_{k l}=l>0$ and suppose that $X_{k l} \rightarrow X_{0}\left[N_{\theta_{r, s}}, M, p\right]^{F}$, and $X_{k l} \rightarrow X_{1}\left[N_{\theta_{r, s}}, M, p\right]^{F}$. Then there exists $\rho_{1}$ and $\rho_{2}$ such that

$$
P-\lim _{r s} \frac{1}{h_{r s}} \sum_{(k, l) \in I_{r, s}}\left(M\left(\frac{d\left(X_{k, l}, X_{0}\right)}{\rho}\right)\right)^{p_{k, l}}=0
$$

and

$$
P-\lim _{r s} \frac{1}{h_{r s}} \sum_{(k, l) \in I_{r, s}}\left(M\left(\frac{d\left(X_{k, l}, X_{1}\right)}{\rho}\right)\right)^{p_{k, l}}=0 .
$$

Let $\rho=\max \left(2 \rho_{1}, 2 \rho_{2}\right)$. Then we have

$$
\begin{aligned}
\frac{1}{h_{r s}} \sum_{(k, l) \in I_{r, s}}\left(M\left(\frac{d\left(X_{1}, X_{0}\right)}{\rho}\right)\right)^{p_{k, l}} & \leq \frac{C}{h_{r s}} \sum_{(k, l) \in I_{r, s}}\left(M\left(\frac{d\left(X_{k, l}, X_{0}\right)}{\rho}\right)\right)^{p_{k, l}} \\
& +\frac{C}{h_{r s}} \sum_{(k, l) \in I_{r, s}}\left(M\left(\frac{d\left(X_{k, l}, X_{1}\right)}{\rho}\right)\right)^{p_{k, l}} \rightarrow 0, \quad(r, s \rightarrow \infty)
\end{aligned}
$$

where $\sup p_{k l}=H, C=\max \left(1,2^{H-1}\right)$. Thus

$$
P-\lim _{r, s} \frac{1}{h_{r s}} \sum_{(k, l) \in I_{r, s}}\left(M\left(\frac{d\left(X_{1}, X_{0}\right)}{\rho}\right)\right)^{p_{k, l}}=0 .
$$

Also, since clearly

$$
P-\lim _{k l}\left(M\left(\frac{d\left(X_{1}, X_{0}\right)}{\rho}\right)\right)^{p_{k, l}}=\left(M\left(\frac{d\left(X_{1}, X_{0}\right)}{\rho}\right)\right)^{l}=0 .
$$

We have

$$
\left(M\left(\frac{d\left(X_{1}, X_{0}\right)}{\rho}\right)\right)^{l}=0 .
$$

Finally we get $X_{0}=X_{1}$. This completes the proof.

Theorem 2.2. Let $\theta_{r, s}=\left\{k_{r}, l_{s}\right\}$ be a double lacunary sequence with $\liminf _{r} q_{r}>1$, and $\liminf _{s} \bar{q}_{s}>1$ then for any Orlicz function $M,\left[\sigma_{1,1}, M, p\right]^{F} \subset\left[N_{\theta_{r, s}}, M, p\right]^{F}$.

Proof. It is sufficient to show that $\left[\sigma_{1,1}, M, p\right]_{0}^{F} \subset\left[N_{\theta_{r, s}}, M, p\right]_{0}^{F}$. The general inclusion follows by linearity. Suppose $\liminf _{r} q_{r}>1$, and $\liminf _{s} \bar{q}_{s}>1$, then there exists $\delta>0$ 
such that $q_{r}>1+\delta$ and $\bar{q}_{s}>1+\delta$. This implies $\frac{h_{r}}{k_{r}} \geq \frac{\delta}{1+\delta}$ and $\frac{\bar{h}_{s}}{l_{s}} \geq \frac{\delta}{1+\delta}$. Then for

$$
\begin{aligned}
& X \in\left[\sigma_{1,1}, M,\right.p]_{0}^{F}, \\
& A_{r, s}= \frac{1}{h_{r s}} \sum_{(k, l) \in I_{r, s}}\left[M\left(\frac{d\left(X_{k, l}, \overline{0}\right)}{\rho}\right)\right]^{p_{k, l}} \\
&= \frac{1}{h_{r s}} \sum_{k=1}^{k_{r}} \sum_{l=1}^{l_{s}}\left[M\left(\frac{d\left(X_{k, l}, \overline{0}\right)}{\rho}\right)\right]^{p_{k, l}} \\
&- \frac{1}{h_{r s}} \sum_{k=1}^{k_{r-1}} \sum_{l=1}^{l_{s-1}}\left[M\left(\frac{d\left(X_{k, l}, \overline{0}\right)}{\rho}\right)\right]^{p_{k, l}} \\
&-\frac{1}{h_{r s}} \sum_{k=k_{r-1}+1}^{k_{r}-1} \sum_{l=1}^{l_{s-1}}\left[M\left(\frac{d\left(X_{k, l}, \overline{0}\right)}{\rho}\right)\right]^{p_{k, l}} \\
&- \frac{1}{h_{r s}} \sum_{l=l_{s-1}+1}^{l_{s}-1} \sum_{k=1}^{k_{r-1}}\left[M\left(\frac{d\left(X_{k, l}, \overline{0}\right)}{\rho}\right)\right]^{p_{k, l}} \\
&= \frac{k_{r} k_{s}}{h_{r s}}\left(\frac{1}{k_{r} l_{s}} \sum_{k=1}^{k_{r}} \sum_{l=1}^{l_{s}}\left[M\left(\frac{d\left(X_{k, l}, \overline{0}\right)}{\rho}\right)\right]^{p_{k, l}}\right) \\
&-\frac{k_{r-1} l_{s-1}}{h_{r s}}\left(\frac{1}{k_{r-1} l_{s-1}} \sum_{k=1}^{k_{r-1}} \sum_{l=1}^{l_{s-1}}\left[M\left(\frac{d\left(X_{k, l}, \overline{0}\right)}{\rho}\right)\right]^{p_{k, l}}\right) \\
&-\frac{1}{h_{r}} \sum_{k=k_{r-1}+1}^{k_{r}-1} \frac{1}{h_{s}} \sum_{l=l_{s-1}+1}^{l_{s}-1} \frac{l_{s}-1}{h_{s}} \frac{1}{l_{s}-1} \sum_{l=1}^{l_{s}-1}\left[M\left(\frac{d\left(X_{k, l}, \overline{0}\right)}{\rho}\right)\right]^{k_{r, l}} \frac{1}{k_{r-1}} \sum_{k=1}^{k_{r-1}}\left[M\left(\frac{d\left(X_{k, l}, \overline{0}\right)}{\rho}\right)\right]^{p_{k, l}} . \\
& k_{k=1}
\end{aligned}
$$

Since $X \in\left[\sigma_{1,1}, M, p\right]^{F}$ the last two terms trends to zero in the Pringsheim sense, thus

$$
\begin{aligned}
& A_{r, s}=\frac{k_{r} k_{s}}{h_{r s}}\left(\frac{1}{k_{r} l_{s}} \sum_{k=1}^{k_{r}} \sum_{l=1}^{l_{s}}\left[M\left(\frac{d\left(X_{k, l}, \overline{0}\right)}{\rho}\right)\right]^{p_{k, l}}\right) \\
& \quad-\frac{k_{r-1} l_{s-1}}{h_{r s}}\left(\frac{1}{k_{r-1} l_{s-1}} \sum_{k=1}^{k_{r-1}} \sum_{l=1}^{l_{s-1}}\left[M\left(\frac{d\left(X_{k, l}, \overline{0}\right)}{\rho}\right)\right]^{p_{k, l}}\right)+o(1) .
\end{aligned}
$$

Since $h_{r s}=k_{r} l_{s}-k_{r-1} l_{s-1}$ we are granted the following:

Observe that

$$
\frac{k_{r} l_{s}}{h_{r s}} \leq \frac{1+\delta}{\delta} \text { and } \frac{k_{r-1} l_{s-1}}{h_{r s}} \leq \frac{1}{\delta} \text {. }
$$

$$
\frac{1}{k_{r} l_{s}} \sum_{k=1}^{k_{r}} \sum_{l=1}^{l_{s}}\left[M\left(\frac{d\left(X_{k, l}, \overline{0}\right)}{\rho}\right)\right]^{p_{k, l}}
$$

and 


$$
\frac{1}{k_{r-1} l_{s-1}} \sum_{k=1}^{k_{r-1}} \sum_{l=1}^{l_{s-1}}\left[M\left(\frac{d\left(X_{k, l}, \overline{0}\right)}{\rho}\right)\right]^{p_{k, l}}
$$

are both Pringsheim null sequences. Thus $A_{r, s}$ is a Pringsheim null sequence. Thus $X$ is in $\left[N_{\theta_{r, s}}, M, p\right]_{0}^{F}$. This completes the proof of this theorem.

Theorem 2.3. Let $\theta_{r, s}=\left\{k_{r}, l_{s}\right\}$ be a double lacunary sequence with $\lim \sup _{r} q_{r}<\infty$, and $\limsup \bar{s}_{r} \bar{q}_{r}<\infty$ then for any Orlicz function $M,\left[N_{\theta_{r, s}}, M, p\right]^{F} \subset\left[\sigma_{1,1}, M, p\right]^{F}$. Proof. Since $\limsup { }_{r} q_{r}<\infty$, and $\limsup _{r} \bar{q}_{r}<\infty$ there exists $H>0$ such that $q_{r}<H$ and $\bar{q}_{s}<H$ for all $r$ and $s$. Let $X \in\left[N_{\theta_{r, s}}, M, p\right]$ and $\varepsilon>0$. Also there exist $r_{0}>0$ and $s_{0}>0$ such that for every $i \geq r_{0}$, and $j \geq s_{0}$

$$
A_{i, j}=\frac{1}{h_{r s}} \sum_{(k, l) \in I_{r, s}}\left[M\left(\frac{d\left(X_{k, l}, \overline{0}\right)}{\rho}\right)\right]^{p_{k, l}}<\varepsilon .
$$

Let $M=\max \left\{A_{i, j}: 1 \leq r \leq r_{0}\right.$ and $\left.1 \leq s \leq s_{0}\right\}, m$ and $n$ be such that $k_{r-1}<m \leq k_{r}$ and $l_{s-1}<n \leq l_{s}$. Then it follows:

$$
\begin{aligned}
& \frac{1}{m n} \sum_{k, l=1,1}^{m, n}\left[M\left(\frac{d\left(X_{k, l}, \overline{0}\right)}{\rho}\right)\right]^{p_{k, l}} \leq \frac{1}{k_{r-1} l_{s-1}} \sum_{k, l=1,1}^{k_{r} l_{s}}\left[M\left(\frac{d\left(X_{k, l}, \overline{0}\right)}{\rho}\right)\right]^{p_{k, l}} \\
& \leq \frac{1}{k_{r-1} l_{s-1}} \sum_{p, u=1,1}^{r, s}\left(\sum_{k, l \in I_{p, u}}\left[M\left(\frac{d\left(X_{k, l}, \overline{0}\right)}{\rho}\right)\right]^{p_{k, l}}\right) \\
& =\frac{1}{k_{r-1} l_{s-1}} \sum_{p, u=1,1}^{r_{0}, s_{0}} h_{p, u} A_{p, u}+\frac{1}{k_{r-1} l_{s-1}} \sum_{\left(r_{0}<p \leq r\right) \cup\left(s_{0}<u \leq s\right)} h_{p, u} A_{p, q} \\
& \leq \frac{M}{k_{r-1} l_{s-1}} \sum_{p, u=1,1}^{r_{0}, s_{0}} h_{p, u}+\frac{1}{k_{r-1} l_{s-1}} \sum_{\left(r_{0}<p \leq r\right) \cup\left(s_{0}<u \leq s\right)} h_{p, u} A_{p, q} \\
& \leq \frac{M k_{r_{0}} l_{s} r_{0} s_{0}}{k_{r-1} s_{s-1}}+\frac{1}{k_{r-1} l_{s-1}} \sum_{\left(r_{0}<p \leq r\right) \cup\left(s_{0}<u \leq s\right)} h_{p, u} A_{p, u} \\
& \leq \frac{M k_{p_{0}} l_{s 0} r_{0} s_{0}}{k_{r-1} l_{s-1}}+\left(\sup _{\left(p \geq r_{0}\right) \cup\left(u \geq s_{0}\right)} A_{p, u}\right) \frac{1}{k_{r-1} l_{s-1}} \sum_{\left(r_{0}<p \leq r\right) \cup\left(s_{0}<u \leq s\right)} h_{p, u} \\
& \leq \frac{M k_{r_{0}} l_{s_{0}} r_{0} s_{0}}{k_{r-1} l_{s-1}}+\frac{1}{k_{r-1} l_{s-1}} \mathcal{E} \sum_{\left(r_{0}<p \leq r\right) \cup\left(s_{0}<u \leq s\right)} A_{p, u} \\
& \leq \frac{M k_{r_{0}} l_{s_{0}} r_{0} s_{0}}{k_{r-1} l_{s-1}}+\varepsilon H^{2} .
\end{aligned}
$$

Since $k_{r}$ and $l_{s}$ both approaches infinity as both $m$ and $n$ approaches infinity. Thus

$$
\frac{1}{m n} \sum_{k, l=1,1}^{m, n}\left[M\left(\frac{d\left(X_{k, l}, \overline{0}\right)}{\rho}\right)\right]^{p_{k, l}} \rightarrow 0
$$


Therefore $X \in\left[\sigma_{1,1}, M, p\right]^{F}$.

The following Theorem is an immediate consequence of Theorem 2.2 and Theorem 2.3

Theorem 2.4. Let $\theta_{r, s}=\left\{k_{r}, l_{s}\right\}$ be a double lacunary sequence with

$$
1<\liminf _{r, s} q_{r, s} \leq \limsup _{r, s} q_{r, s}<\infty,
$$

then for any Orlicz function $M,\left[N_{\theta_{r, s}}, M, p\right]^{F}=\left[\sigma_{1,1}, M, p\right]^{F}$.

Theorem 2.5. Let the sequence $p_{k, l}$ be bounded, then

$$
\left[N_{\theta_{r, s}}, M, p\right]_{0}^{F} \subset\left[N_{\theta_{r, s}}, M, p\right]^{F} \subset\left[N_{\theta_{r, s}}, M, p\right]_{\infty}^{F}
$$

Proof. Let $X$ be element of $\left[N_{\theta_{r, s}}, M, p\right]^{F}$. Then we have

$$
\begin{aligned}
\frac{1}{h_{r s}} \sum_{(k, l) \in I_{r, s}}\left[M\left(\frac{d\left(X_{k, l}, \overline{0}\right)}{2 \rho}\right)\right]^{p_{k, l}} & \leq \frac{C}{h_{r s}} \sum_{(k, l) \in I_{r, s}} \frac{1}{2^{p_{k} k l}}\left[M\left(\frac{d\left(X_{k, l}, \overline{0}\right)}{\rho}\right)\right]^{p_{k, l}} \\
& +\frac{C}{h_{r s}} \sum_{(k, l) \in I_{r, s}} \frac{1}{2^{p_{k l}}}\left[M\left(\frac{d\left(X_{0}, \overline{0}\right)}{\rho}\right)\right]^{p_{k, l}} \\
\leq & \frac{C}{h_{r s}} \sum_{(k, l) \in I_{r, s}} \frac{1}{2^{p_{k l}}}\left[M\left(\frac{d\left(X_{k, l}, X_{0}\right)}{\rho}\right)\right]^{p_{k, l}} \\
& +C \max \left(1, \sup \left[M\left(\frac{d\left(X_{0}, \overline{0}\right)}{\rho}\right)\right]^{H}\right),
\end{aligned}
$$

where $\sup p_{k l}=H$ and $C=\max \left(1,2^{H-1}\right)$. Thus we have $X \in\left[N_{\theta_{r, s}}, M, p\right]_{\infty}^{F}$. The inclusion $\left[N_{\theta_{r, s}}, M, p\right]_{0}^{F} \subset\left[N_{\theta_{r, s}}, M, p\right]^{F}$ is obvious.

In [27], Savas defined lacunary statistical convergence for double sequence of fuzzy numbers as follows:

Definition 2.5. Let $\theta_{r, s}$ be a double lacunary sequence; the double number sequence $X$ is $S_{\theta_{r, s}}-P$-convergent to L provided that for every $\varepsilon>0$,

$$
P-\lim _{r, s} \frac{1}{h_{r, s}} \mid\left\{(j, k) ; j \leq m \text { and } k \leq n: d\left(X_{k l}, X_{0}\right) \geq \varepsilon\right\} \mid=0 .
$$

In this case we write $S_{\theta_{r, s}}(F)-\lim _{k, l} X_{k, l}=X_{0}$ and we denote the set of all double lacunary statistically convergent sequences of fuzzy numbers by $S_{\theta_{r, s}}(F)$. We shall now establish an inclusion theorem between $\left[N_{\theta_{r, s}}, M\right]^{F}$ and $S_{\theta_{r, s}}(F)$.

Theorem 2.6. For any Orlicz function $M,\left[N_{\theta_{r, s}}, M\right]^{F} \subset S_{\theta_{r, s}}(F)$. 
Proof. Let $X \in\left[N_{\theta_{r, s}}, M\right]$ and $\varepsilon>0$. Then

$$
\begin{aligned}
\frac{1}{h_{r s}} \sum_{(k, l) \in I_{r, s}} M\left(\frac{d\left(X_{k, l}, \overline{0}\right)}{\rho}\right) & \geq \frac{1}{h_{r s}} \sum_{(k, l) \in I_{r, s}: d\left(X_{k, l}, X_{0}\right) \geq \varepsilon} M\left(\frac{d\left(X_{k, l}, \overline{0}\right)}{\rho}\right) \\
& >\frac{1}{h_{r s}} M\left(\frac{\varepsilon}{\rho}\right) \frac{1}{h_{r, s}}\left|\left\{(k, l) \in I_{r, s}: d\left(X_{k l}, X_{0}\right) \geq \varepsilon\right\}\right| .
\end{aligned}
$$

Thus $X \in S_{\theta_{r, s}}(F)$.

\section{REFERENCES}

1. L. C. Barros, R. C. Bassanezi and P. A. Tonelli, Fuzzy modelling in population dynamics, Ecol. Model. 128, 27-33, 2000.

2. R. C. Buck, Generalized asymptotic density, American J. Math. 75, 335-346, 1953.

3. P. Diomand and P. Kloeden, Metric spaces of fuzzy sets, Fuzzy Sets and Systems, 33, 123-126, 1989.

4. M. S, El Naschie, On certainty of Cantorian geometry and two-slit experiment, Chaos, Solitons \& Fractals; 9, 517-29, 1998.

5. M. S, El Naschie, On the unification of heterotic strings, $M$-theory and $\varepsilon^{\infty}$-theory. Chaos, Solitons \& Fractals, 11, 2397-408, 2000.

6. M. S, El Naschie, From experimental quantum optics to quantum gravity via fuzzy Kähler manifold, Chaos, Solitons \& Fractals, 25, 969-77, 2005.

7. H. Fast,. Sur la convergence statistique, Colloq. Math. 2, 241-244, 1951.

8. A. L. Fradkov, R. J. Evans, Control of chaos: Methods and applications in engineering, Chaos, Solitons \& Fractals, 29, 33-56, 2005.

9. R. Giles, A computer program for fuzzy reasoning, Fuzzy Sets and System, 4, 221234, 1980.

10. L. Hong and J. Q. Sun, Bifurcations of fuzzy nonlinear dynamical systems, Comm. Non-linear Sci. Numer. Simul. 1, 1- 12, 2006.

11. P. K. Kamthan and M. Gupta, Sequence spaces and series, Marcel Dekker Inc. NewYork, 1981.

12. M. A. Krasnoselskii and Y. B. Rutisky, Convex function and Orlicz spaces, Groningen, Netherlands, 1961.

13. J. Madore, Fuzzy physics, Ann. Phys. 219, 187- 198, 1992.

14. J. Lindenstrauss and L. Tzafriri, On Orlicz sequence spaces, Israel J. Math. 10, 379-390, 1971.

15. A. Gokhan, M. Et and M. Mursaleen, Almost lacunary statistical and strongly almost lacunary convergence of sequences of fuzzy numbers, Math. Comput. Model., 49(3-4), 548-555, 2009.

16. Mursaleen and M. Basarir, On some new sequence of fuzzy numbers, Indian J. Pure Appl. Math. 34 (9), 1351-1357, 2003.

17. Mursaleen and O. H. Edely, Statistical convergence of double sequences, J. Math. Anal. Appl. 288 (1), 223-231, 2003.

18. S. Nanda, On sequence of Fuzzy numbers, Fuzzy Sets and System, 33, 123-126, 1989. 
19. F. Nuray, Lacunary statistical convergence of sequences of fuzzy numbers, Fuzzy Sets and System, 99(3), 353-355, 1998.

20. S. D. Parashar and B. Choudhary, Sequence spaces defined by Orlicz functions, Indian J. Pure Appl. Math., 25(4), 419-428, 1994.

21. A. Pringsheim, Zur theorie der zweifach unendlichen Zahlenfolgen, Mathematische Annalen, 53, 289-321, 1900.

22. R. Saadati, S. Mansour Vaezpour and,Yeol J. Cho, Quicksort algorithm: Application of a fixed point theorem in intuitionistic fuzzy quasi-metric spaces at a domain of words, J. Comput. Appl. Math. 228 (1) , 219 -225, 2009.

23. E. Savas, A note on double sequence of Fuzzy numbers, Turk. J. Math., 20, 175$178,1996$.

24. E. Savas, A note on sequence of Fuzzy numbers, Inform. Sci., 124, 297-300 , 2000.

25. E. Savas, On statistically convergent sequence of Fuzzy numbers, Inform. Sci., 137, 272-282, 2001

26. E. Savas and Mursaleen, On statistically convergent double sequence of Fuzzy numbers, Inform. Sci., 162, 183-192, 2004.

27. E. Savas, On lacunary statistical convergent sequences of fuzzy numbers, Appl. Math. Lett., 21, 134-141, 2008

28. A. Zadeh, Fuzzy Sets, Infor. Control , 8, 338-353, 1965. 